\section{Expression of PADI4 during rheumatoid arthritis development}

\author{
Cuirong Bian, 'Xiaotian Chang, \\ Yan Zhao, ${ }^{2}$ Jinqiu Jia, ${ }^{2}$ Yunzhong Zhang ${ }^{3}$ \\ 'Qilu Hospital of Shandong University, \\ Jinan, Shandong, China; \\ ${ }^{2}$ Research Center for Biotechnology, \\ Shandong Academy of Medical Sciences, \\ Jinan, Shandong, China; ${ }^{3}$ Affiliated \\ Hospital of Shandong Academy of \\ Medical Sciences, Jinan, Shandong, China; \\ ${ }^{4}$ Zhifang Hospital of Shandong, Jinan, \\ Shandong, P. R. China
}

\section{Abstract}

Peptidyl deiminase 4 (PADI4) catalyzes peptidylarginine to citrulline. The gene encoding PADI4 is associated with rheumatoid arthritis (RA) in some populations. The levels of PADI4, anti-CCP, and RF were measured in the blood of patients suffering from RA and osteoarthritis (OA) for less than six months using ELISA. The levels were also measured in the blood of patients that have been diagnosed with RA for more than three years. As controls, samples from healthy subjects or patients with ankylosing spondylitis (AS), epidemic pleurodynia (EP), OA, psoriatic arthritis (PA), systemic lupus erythematosus (SLE), gouty arthritis (GA), or Still's disease (ST) were also assayed. Of patients with early RA, $68.7 \%$ had significantly higher levels of PADI4 than patients with early OA. Patients with RA for a longer period of time had a higher expression of PADI4 than patients with SLE, ST, and GA, but they were similar to healthy subjects or patients with AS, EP, OA, or PA. The level of PADI4 was significantly correlated to the levels of RF and anti-CCP in blood samples of RA, but not in the samples of other diseases and those of healthy subjects. The result suggests that PDI4 play important role for the onset of RA. The level of PADI4 relates to the disease activity and clinical performance.

\section{Introduction}

A functional haplotype of peptidylarginine deiminase 4 (PADI4) gene is susceptible to rheumatoid arthritis (RA) in Japanese, Korean, and German populations. ${ }^{1-3}$ Patients with RA, an autoimmune disease, have diverse autoantibodies in their blood, including anti-filaggrin antibody (AFA), anti-ker- atin antibody (AKA), anti-perinuclear factor (APF), and other autoantibodies. The B-cell epitope for these autoantibodies contains citrulline, an amino acid metabolite of arginine. PADI4 converts peptidylarginine to citrulline in a process known as citrullination. Hence, PADI4 and its citrullination activity are considered to play an essential role in pathogenesis of RA., Although PADI4 has been detected in synovial tissues of RA, ${ }^{6}$ only a few studies have investigated the level of the enzyme in the blood of patients, and there is no report about the expressional profile during the pathogenic process of the disease.

In the present study, we measured the level of PADI4 in the blood of patients with recent RA, i.e., less than six months, as well as in the blood of patients with established RA i.e., over three years. We have also examined the levels of anti-cyclic citrullinated peptide antibodies (anti-CCP) and rheumatoid factors (RF) in the samples. Anti-CCP and RF have high sensitivity and specificity for RA and have been related to the progression of joint damage. ${ }^{7}$ By comparing the expressions of PADI4, RF and anti-CCP in the blood from patients of RA at different pathogenic stages, we aimed to investigate the relation of PADI4 expression with the clinical performance of the disease.

\section{Materials and Methods}

\section{Blood samples}

Blood samples were obtained by standard venepuncture from patients with RA $(n=617$; 511 female; 13-81 years old, mean 49 years), ankylosing spondylitis (AS, $\mathrm{n}=52 ; 12$ female; 14-58 years old, mean 29 years), epidemic pleurodynia (EP, $n=49$; 16 female; $20-58$ years old, mean 42 years), osteoarthritis ( $0 \mathrm{~A}, \mathrm{n}=49$; 33 female; 43-71 years old, mean 57 ), psoriatic arthritis (PA, $n=49 ; 15$ female; 32-51 years old, mean 44 years), systemic lupus erythematosus (SLE, $n=44,37$ female; 8-60 years old, mean 29 years), gouty arthritis (GA, $n=45$; 9 female; $37-66$ years old, mean 50 years), and Still's disease (ST, $\mathrm{n}=44 ; 12$ female, 8 - 14 years old, mean 10 years). One hundred and sixty blood samples from healthy volunteers (93 female; 19-58 years old, mean 35 years) were used as controls. Patients with RA fulfilled the American College of Rheumatology 1987 revised criteria (ACR) and had the disease for over three years. The samples were put into Monovette tubes containing $3.8 \%$ sodium citrate. Following centrifugation at $1000 \mathrm{X}$ g for $30 \mathrm{~min}$, the supernatant of anticoagulated plasma was collected and stored at $-80^{\circ} \mathrm{C}$ until using.

In addition, blood samples were obtained
Correspondence: Xiaotian Chang,

Jiangshi Road 89, Jinan, Shandong, 250062, P. R.

China. E-mail: changxt@sdams.cn

Key words: rheumatoid arthritis, peptidylarginine deiminase 4 , citrullination, anti-cyclic citrullinated peptide antibodies, rheumatoid factors.

Acknowledgments: the present study was supported by the National Natural Science Foundation of China (NTFC) (30671949), the Shandong Scientific Instrument Equipment Promotion Transformation Project (2006GG1108097-41, 2007GG2TC02050) and the Leading Project of Medical Science of Shandong.

Competing interests: the authors declare that they have no competing interests.

Contributions: XC, designed the study and prepared the manuscript; CB and YZ, performed experiments; JJ and YZ, collected blood samples.

Received for publication: 22 April 2009.

Revision received: 19 May 2009.

Accepted for publication: 3 July 2009.

This work is licensed under a Creative Commons Attribution 3.0 License (by-nc 3.0)

(C) Copyright C. Bian et al., 2009

Licensee PAGEPress, Italy

Rheumatology Reports 2009; 1:e3

doi:10.4081/rr.2009.e3

from 88 patients who had arthritis of one or poly joints for 1-6 months when they visited clinics for the first time. After 1-2 year followup, the 48 patients with undifferentiated arthritis (UA) were diagnosed with RA (35 female, 32-63 years old, mean 41 years), and the other 40 patients were diagnosed with $\mathrm{OA}$ (27 female, 45-69 years old, mean 54 years). Fifty blood samples were selected from the healthy group who were matched for sex and age to those of the experimental group.

In the present study, all patients and healthy volunteers provided a written, informed consent, and the ethics committee of Shandong Academy of Medical Science approved the protocol of the study.

\section{ELISA}

The level of PADI4 in blood samples was measured using ELISA. The blood sample was diluted 20 -fold in $0.05 \mathrm{M}$ carbonate-bicarbonate buffer $(\mathrm{pH} \mathrm{9.6)}$ and coated onto 96-well EIA/RIA microtiter plates (Costar) by incubation at $4^{\circ} \mathrm{C}$ overnight. After a brief washing with PBS ( $8 \mathrm{~g} \mathrm{NaCl}, 0.2 \mathrm{~g} \mathrm{KCl}, 1.15 \mathrm{~g} \mathrm{NaHPO} 4$ and $0.2 \mathrm{~g} \mathrm{KH} 2 \mathrm{PO} 4$ per liter, pH 7.4-7.6) containing $0.1 \%$ Tween 20 (PBST), the plate was blocked with $5 \%$ non-fat dry milk for $1 \mathrm{~h}$ at room temperature. The anti PADI4 antibody was diluted 4000 -fold with PBST, added onto 
the plate, and incubated for $2 \mathrm{~h}$ at room temperature. Immunizing rabbits with synthetic oligo-peptide (FGDSCYPSNDSRQMH) against PADI4 amino acid sequence gave the antibody. The immunospecificity of the antibody had been confirmed in our previous study. ${ }^{6}$ After washing with PBST, the plate was incubated with 10,000-fold dilution of Anti-Rabbit IgG Alkaline Phosphatase Conjugate Antibody (Sigma) for $30 \mathrm{~min}$ at room temperature. After washing with PBST, the signal was developed by adding Alkline Phosphatase Yellow (pNPP) liquid Substrate System for ELISA (Sigma). The absorbance of the reaction was measured at $405 \mathrm{~nm}$ with a plate reader (Synergy HT, Bio-Tek). Negative controls consisted of wells with carbonatebicarbonate buffer instead of blood samples (antibody non-specific binding) and wells with PBST buffer instead of antibody (sample non-specific binding). Data of the experiment were corrected by subtracting the sum of the non-specific binding and the antibody non-specific binding.

0.D. values of PADI4 of healthy subjects were averaged and treated as reference for the experiment. Levels of PADI4 in all samples were calculated by dividing their 0.D. values with the average. In this way, PADI4 levels of all samples were expressed with relative unit.

\section{Measurement of anti-CCP and RF}

ImmunoScan RA Anti-CCP Test Kit was commercially obtained from EuroDiagnostica. Anti-CCP level in the blood was measured by ELISA according to the manufacturer's instructions. The samples were diluted 10 times with dilution buffer (provided by the manufacturer) before the measurement. Anti-CCP value above $50 \mathrm{IU} / \mathrm{mL}$ was considered positive according to the manufacturer's protocol. RF level was measured by immunonephelometry with BN ProSpec ${ }^{\circledR}$ (Dade Behring, USA), a system performing routine plasma $\mathrm{RF}$ assays using $\mathrm{N}$ Latex RF kit (Dade Behring, USA) that specially detects IgM RF. An RF value above $25 \mathrm{U}$ was considered positive according to the manufacturer's protocol. To ensure a correct diagnosis, all samples of SLE we obtained from the clinical laboratory were negative with RF.

Data of the previously described ELISA were collected from three independent tests. Statistical analyses were conducted using SPSS (version 11.0). The levels of PADI4, RF and anti-CCP in the blood samples were expressed as median and range. The differences between groups were statistically assessed by the Mann-Whitney U-test. The correlations among PADI4, RF and anti-CCP were analyzed by Spearman's non-parametric rank correlation. A level of $p<0.05$ was considered significant.

\section{Results}

Level of PADI4 in blood samples of UA was measured by ELISA. The mean level of PADI4 for the healthy group was $1.0024 \pm 0.4472$ relative unit. Forty-eight of the 88 patients with UA were finally diagnosed with RA in 1-2 years. The mean level of PADI4 for the very early RA was $2.742 \pm 1.2882$ relative unit. Thirty-three of the 48 samples (68.7\%) from the recent-onset RA expressed the highest levels of PADI4 ( $>2$ relative unit) among all UA samples, whereas 20 of them also expressed high levels of anti$\mathrm{CCP}$ (> $50 \mathrm{IU} / \mathrm{mL})$, and 11 showed high values
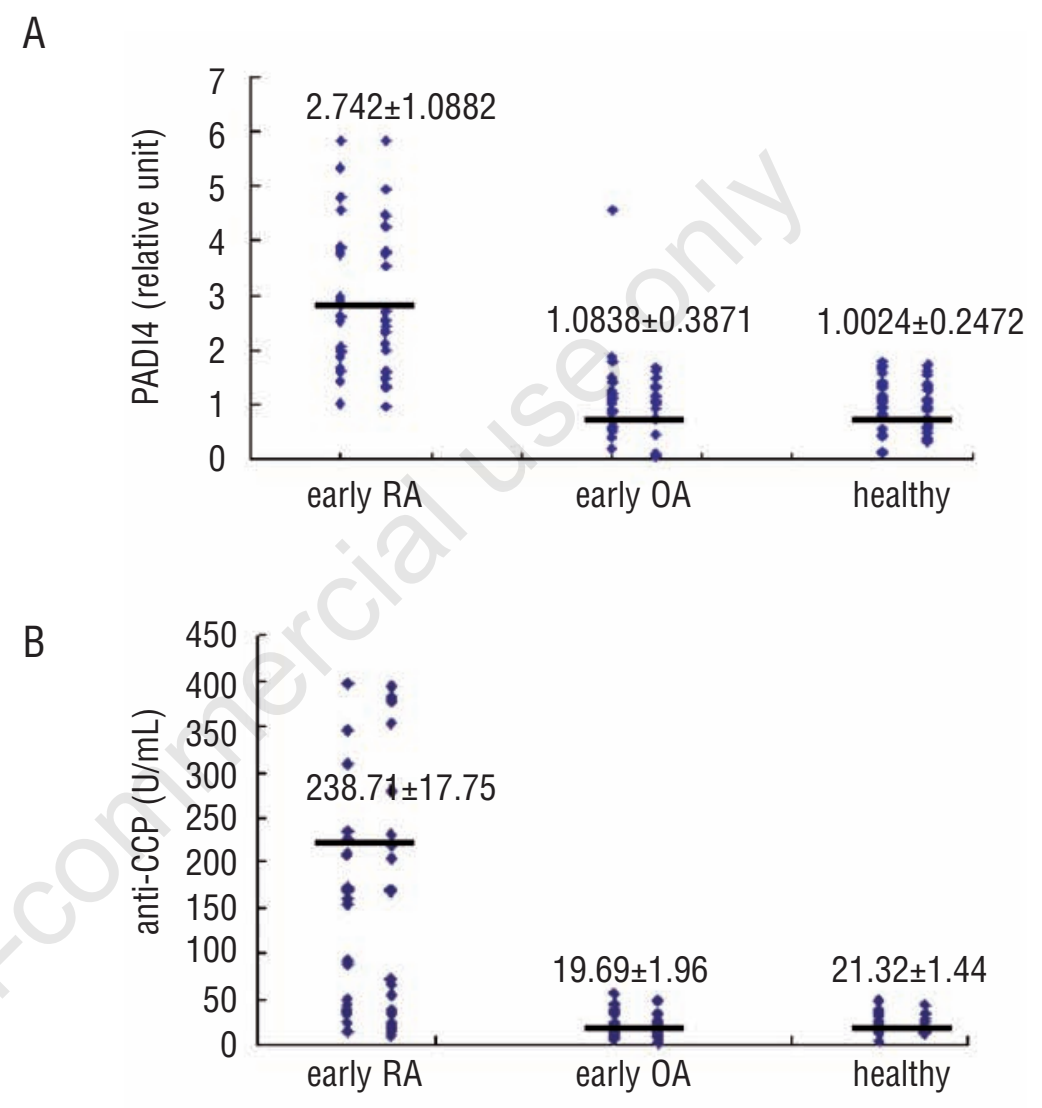

C

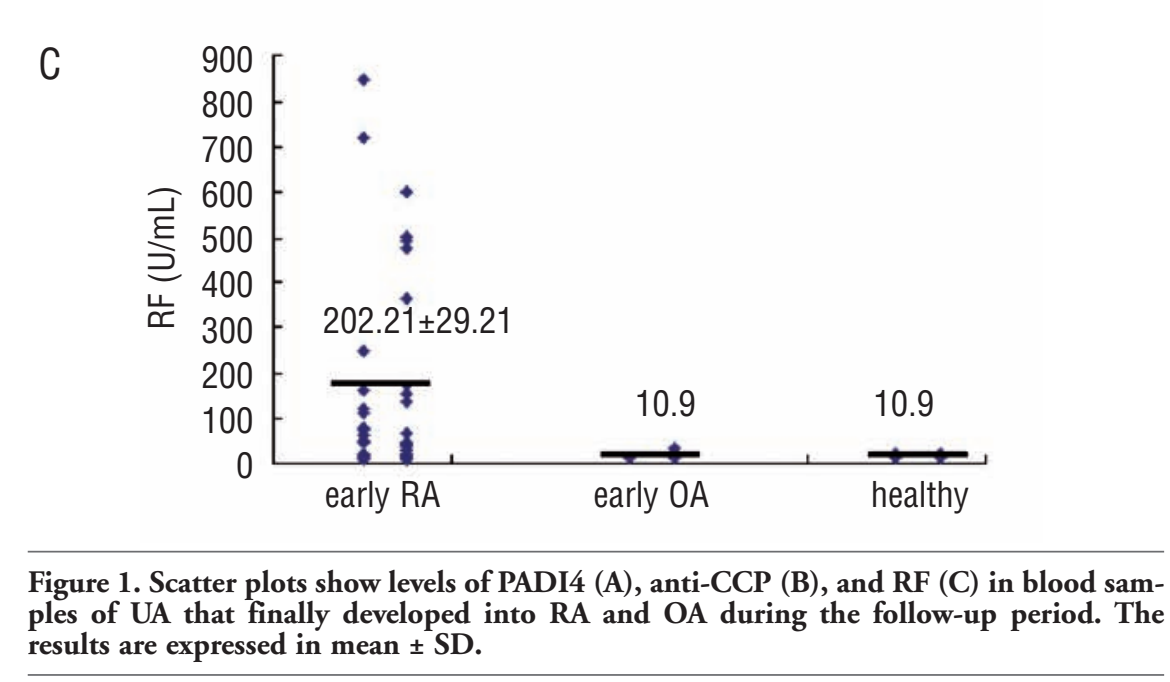

Figure 1. Scatter plots show levels of PADI4 (A), anti-CCP (B), and RF (C) in blood samples of UA that finally developed into RA and OA during the follow-up period. The results are expressed in mean $\pm S D$. of $\mathrm{RF}$ ( $>25 \mathrm{IU} / \mathrm{mL}$ ). The mean level of PADI4 for early $\mathrm{OA}$ was $1.0838 \pm 0.3871$ relative unit, which was similar to the level of the healthy group. Five patients (11.3\%) who finally showed symptoms of OA during the follow-up period expressed PADI4 at relatively high levels (1.2-2 relative unit). Eight of the 50 samples (16\%) of the healthy controls expressed PADI4 at levels of 1.2-2 relative unit. The expression of PADI4 was significantly correlated with the expressions of RF ( $p=0.011)$ and anti-CCP $(p=0.008)$ in all UA samples. The above results are shown in Figure 1. If the PADI4 level $\geq 2$ relative unit was considered positive, the positive rate of RA (68.7\%) is sig-

\section{B}


nificantly higher than positive rate of $\mathrm{OA}$ (2.5\%) and the healthy group (0).

In another ELISA experiment, levels of PADI4, RF, and anti-CCP were measured in blood samples of patients with established RA. Samples from patients with other arthritic diseases and autoimmune diseases were used as controls. The PADI4 level of the established RA was similar to the levels of AS, EP, OA, PA, and the healthy group, but higher than the levels of SLE, ST, and GA. If the PADI4 level $\geq 2$ relative unit was considered positive, the positive rates of RA, AS and OA are similar (around 8\%-10\%) and higher than positive rates of other diseases and the healthy group (around 0-2\%). The PADI4 level was significantly correlated with the levels of RF ( $p=0.0001)$ and anti-CCP $(p=0.02)$ in samples of RA but not in samples of other diseases and the healthy group. The above results are shown in Figure 2.

To test the reliability of the above study, levels of some other proteins such as Hsp 70 and retinal dehydrogenase 1 (ALDH1A1) in the blood samples were measured using rabbit polyclonal antibodies and monoclonal antibodies by the same ELISA protocol. There were no significant associations between the levels of these proteins and the levels of anti-CCP or RF. The level of PADI4 was also measured with monoclonal antibody and polyclonal antibody produced by abcam (catalog number: ab59965, ab50332 and ab57167). The results of ELISA were very similar to the results obtained with our polyclonal antibody. These experiments confirmed the measurement of PADI4 and its association with anti-CCP and RF.

\section{Discussion}

Cha et al. have recently confirmed the relationship between anti-CCP levels and PADI4 haplotypes by analyzing 311 patients with nonerosive and erosive RA. They have found that anti-CCP levels were significantly higher in patients carrying the PADI4 RA risk haplotype than in patients who did not. ${ }^{8}$ They have also discovered that the PADI4 RA risk haplotype was associated with increased anti-CCP levels in patients with early RA. This suggests that the expression of PADI4 essentially affects the early progress of RA. In the present study, over $90 \%$ of patients at an early stage of RA had relatively high levels ( $>1.2$ relative unit) of PADI4 in their blood, and $70 \%$ of them expressed much higher levels of PADI4 than patients with other UA or healthy patients. Verpoort et al. have also detected IgM RF and anti-CCP in samples of early RA patients. ${ }^{9}$ Nissinen R et al. have reported increased levels of IgG antibodies to $\mathrm{PAD}$ in $88 \%$ of recent-onset RA patients and in $70 \%$ of patients that have been diagnosed for three years. ${ }^{10}$ On the other hand, we

A

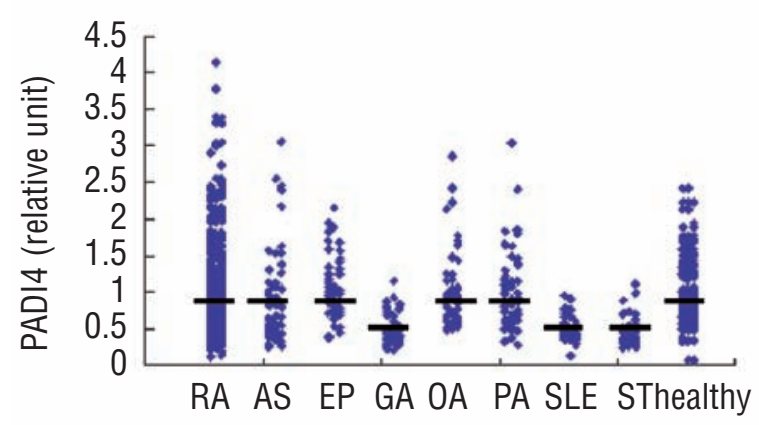

B

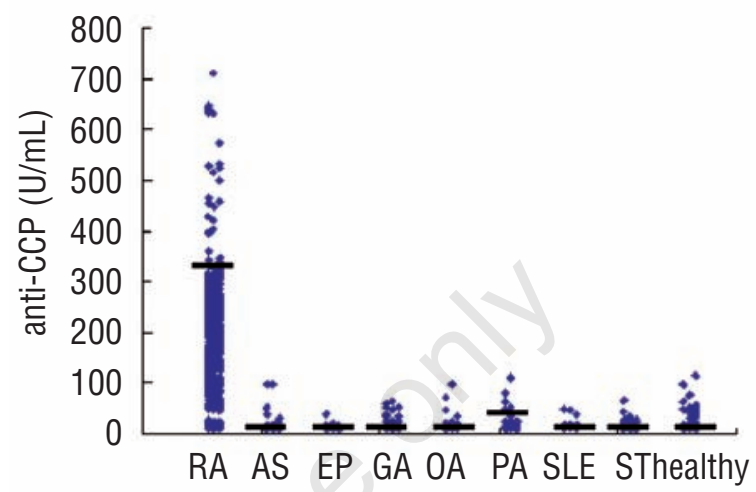

C

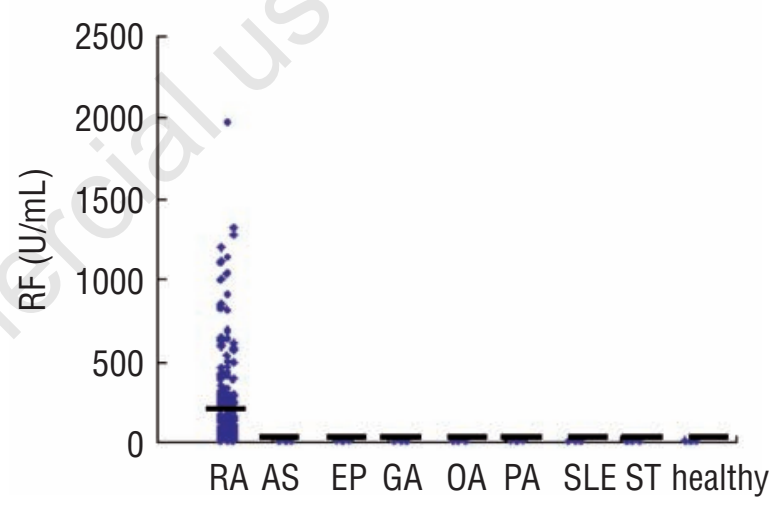

D

\begin{tabular}{lccc} 
Disease & PADI4 (0.D.) & anti-CCP IU/mL & RF IU/mL \\
AS $(n=52)$ & $0.3726 \pm 0.0307$ & $17.65 \pm 2.49$ & 10.9 \\
EP $(n=49)$ & $0.4144 \pm 0.0245$ & $13.82 \pm 0.6139$ & 10.9 \\
\hline GA $(n=45)$ & $0.1921 \pm 0.0123$ & $21.82 \pm 2.09$ & 10.9 \\
OA $(n=48)$ & $0.4037 \pm 0.0316$ & $17.80 \pm 2.25$ & 10.9 \\
\hline PA $(n=49)$ & $0.4053 \pm 0.0318$ & $21.02 \pm 2.81$ & 10.9 \\
RA ( $n=627)$ & $0.3923 \pm 0.0105$ & $346.94 \pm 24.95$ & $90.57 \pm 7.83$ \\
\hline SLE $(n=44)$ & $0.1972 \pm 0.01$ & $16.78 \pm 1.26$ & 10.9 \\
ST $(n=44)$ & $0.1817 \pm 0.0121$ & $18.52 \pm 1.54$ & 10.9 \\
\hline health $(n=160)$ & $0.40342 \pm 0.0163$ & $17.15 \pm 1.204$ & 10.9
\end{tabular}

Figure 2. Scatter plots show the levels of PADI4 (A), anti-CCP (B), and RF (C) in blood samples from various arthritis, autoimmune diseases, and the healthy controls. The samples of RA were obtained from patients with the disease for more than three years. The contents of PADI4, anti-CCP, and RF are shown in the table in panel D and are expressed in mean \pm SD.

have found that PADI4 was not significantly elevated in the samples of the established RA as compared to the healthy group and some of the other diseases that we have tested. To put the results of these studies together, we suggest that PADI4 increases its expression at an 
early stage of RA, which might break immunological tolerance by catalyzing synovial proteins and consequently stimulates the pathogenic process of the disease. Lundberg et al. have found that collagen II following citrullination induced arthritis with earlier onset and had higher incidence than unmodified collagen to immunize rats. ${ }^{11}$

Bartone et al. have reported that PADI4 gene polymorphisms were not associated with severity and anti-CCP by analyzing RA patients with erosive outcome for five years. ${ }^{12}$ Cha et al. have also found that PADI4 haplotype was not associated with anti-CCP and RF among patients who had the disease for a longer period of time. ${ }^{8}$ In our study, the mean level of PADI4 protein was not elevated in the blood of the established RA as compared to the levels of other arthritis including OA, PA, and ST. This is in agreement with the findings of others. These results support that PADI4 and synovial citrullinated products are not specific for the pathogenesis of RA in a long-standing stage and that citrullination appears to be a general process for joint inflammation in arthritic mice and humans. ${ }^{11}$ On the other hand, our study has especially detected significant correlations between PADI4 and RF and between PADI4 and anti-CCP in RA blood, indicating that PADI4 and its citrullination activity are related to an autoimmune reaction when RA progresses into a more severe and chronic state. Takizawa et al. have detected a significantly higher prevalence of anti-PADI4 in 50\% RA patients by ELISA using recombinant PADI4 protein, suggesting that PADI4 is an autoantigen in some RA patients. ${ }^{12}$

To summarize, we have found that PADI4 had a high expression at a very early stage of RA but was expressed at normal levels when RA progressed into a chronic stage. This sug- gests that PADI4 possibly plays an important role for the onset of RA. RF and anti-CCP were strongly associated with joint erosions and deformities of the disease. ${ }^{7,13}$ Since the PADI4 level was significantly associated with the levels of RF and anti-CCP in the blood of RA patients at early and established stages, the expression of the enzyme possibly relates to the disease activity and clinical performance.

\section{References}

1. Suzuki A, et al. Functional haplotypes of PADI4, encoding citrullinating enzyme peptidylarginine deiminase 4 , are associated with rheumatoid arthritis. Nat Genet 2003;34:395-402.

2. Kang CP, Lee HS, Ju H, Cho H, Kang C, Bae SC. A functional haplotype of the PADI4 gene associated with increased rheumatoid arthritis susceptibility in Koreans. Arthritis Rheum 2006;54:90-6.

3. Hoppe B, Haupl T, Gruber R, et al. Detailed analysis of the variability of peptidylarginine deiminase type 4 in German patients with rheumatoid arthritis: a case-control study. Arthritis Res Ther 2006;8:R34-6.

4. Van Venrooij WJ, Pruijn, GJ. Citrullination: a small change for a protein with great consequences for rheumatoid arthritis. Arthritis Res 2000;2:249-51.

5. Utz PJ, Genovese MC, Robinson WH. Unlocking the "PAD" lock on rheumatoid arthritis. Ann Rheum Dis 2004;63:330-2.

6. Chang X, Yamada R, Suzuki A, et al. Localization of peptidylarginine deiminase 4 (PADI4) and citrullinated protein in synovial tissue of rheumatoid arthritis. Rheumatology (Oxford) 2005;44:40-50.
7. Agrawal S, Misra R, Aggarwal A. Autoantibodies in rheumatoid arthritis: association with severity of disease in established RA. Clin Rheumatol 2007;26: 201-4.

8. Cha S, Choi CB, Han TU, et al. Association of anti-cyclic citrullinated peptide antibody levels with PADI4 haplotypes in early rheumatoid arthritis and with shared epitope alleles in very late rheumatoid arthritis. Arthritis Rheum 2007;56:1454-63.

9. Verpoort KN, et al. Isotype distribution of anti-cyclic citrullinated peptide antibodies in undifferentiated arthritis and rheumatoid arthritis reflects an ongoing immune response. Arthritis Rheum 2006; 54: 3799808.

10. Nissinen R, Paimela L, Julkunen H, et al. Peptidylarginine deiminase, the arginine to citrulline converting enzyme, is frequently recognized by sera of patients with rheumatoid arthritis, systemic lupus erythematosus and primary Sjögren syndrome. Scand J Rheumatol 2003;32:33742.

11. Lundberg K, Nijenhuis S, Vossenaar ER, et al. Citrullinated proteins have increased immunogenicity and arthritogenicity and their presence in arthritic joints correlates with disease severity. Arthritis Res 2005; 7:R458-R67.

12. Takizawa Y, Sawada T, Suzuki A, et al. Peptidylarginine deiminase 4 (PADI4) identified as a conformation-dependent autoantigen in rheumatoid arthritis. Scand J Rheumatol 2005;34:212-5.

13. Forslind K, Ahlmén M, Eberhardt K, et al. Prediction of radiological outcome in early rheumatoid arthritis in clinical practice: role of antibodies to citrullinated peptides (anti-CCP). Ann Rheum Dis 2004;63:1090-5. 